Case Report

\title{
Globus Pallidus Internus Deep Brain Stimulation for Disabling Diabetic Hemiballism/Hemichorea
}

\author{
Byung-chul Son, ${ }^{1,2}$ Jin-gyu Choi, ${ }^{1}$ and Hak-cheol Ko ${ }^{1}$ \\ ${ }^{1}$ Department of Neurosurgery, Seoul St. Mary's Hospital, College of Medicine, The Catholic University of Korea, \\ Seoul, Republic of Korea \\ ${ }^{2}$ Catholic Neuroscience Institute, College of Medicine, The Catholic University of Korea, Seoul, Republic of Korea \\ Correspondence should be addressed to Byung-chul Son; sbc@catholic.ac.kr
}

Received 22 April 2017; Revised 3 July 2017; Accepted 19 September 2017; Published 23 October 2017

Academic Editor: Mathias Toft

Copyright (C) 2017 Byung-chul Son et al. This is an open access article distributed under the Creative Commons Attribution License, which permits unrestricted use, distribution, and reproduction in any medium, provided the original work is properly cited.

\begin{abstract}
Unilateral hemichorea/hemiballism (HH) associated with contralateral neuroimaging abnormalities of the basal ganglia, which is characterized by $\mathrm{T} 1$ hyperintensity on magnetic resonance imaging (MRI) and is secondary to diabetic nonketotic hyperglycemia, is a rare and unique complication of poorly controlled diabetes mellitus (DM). Although almost all prior reports have documented rapid resolution of $\mathrm{HH}$ within days after normalization of blood glucose levels, medically refractory persistent $\mathrm{HH}$ has been noted. The experience of surgical intervention for persistent $\mathrm{HH}$ is limited. A 46-year-old, right-handed female patient with type $2 \mathrm{DM}$ presented with refractory diabetic $\mathrm{HH}$ on the left side of 6 months' duration despite DM control and neuroleptic medication usage. Image-guided deep brain stimulation (DBS) on the right globus pallidus internus (GPi) was performed. A mechanical micropallidotomy effect was observed and chronic stimulation of GPi was quite effective in symptomatic control of diabetic $\mathrm{HH}$ until a 16-month follow-up visit. DBS of the GPi can be an effective treatment for medically refractory diabetic HH.
\end{abstract}

\section{Introduction}

Hemichorea/hemiballism (HH) is a hyperkinetic movement disorder characterized by acute or subacute onset of highamplitude, involuntary movements affecting one side of the body $[1,2]$. Ischemic/hemorrhagic stroke within the contralateral subthalamic nucleus (STN) and basal ganglia and nonketotic hyperglycemia are the most common causes of $\mathrm{HH}$. Diabetic HH is a unique syndrome characterized by $\mathrm{HH}$, hyperglycemia, and striatal hyperintensity on T1-weighted magnetic resonance image (MRI) [3].

The clinical course of diabetic $\mathrm{HH}$ is favorable and the symptoms tend to resolve rapidly with normalization of hyperglycemia in patients who develop diabetic $\mathrm{HH}$ secondary to nonketotic hyperglycemia [2, 4]. However, there are rare patients who continue to have persistent $\mathrm{HH}$ years after the inciting hyperglycemic crisis [5]. In rare, medically refractory diabetic $\mathrm{HH}$, stereotactic lesioning of the ventrolateral (VL) thalamus [6] and GPi [7] and DBS of the thalamic ventralis oralis (VO) nucleus [8] have been reported to show significant benefit in case reports. Although
GPi DBS was reported to be effective in a patient with $\mathrm{HH}$ secondary to vascular insult to the STN and substantia nigra [9], its effectiveness in diabetic $\mathrm{HH}$ has not yet been reported. We report the effectiveness of chronic GPi stimulation in a patient suffering refractory diabetic $\mathrm{HH}$ and summarized the utility of stereotactic surgery in this rare hyperkinetic movement disorder of metabolic cause.

\section{Case Presentation}

A 46-year-old, right-handed female patient with an 11-year history of type 2 diabetes mellitus (DM) presented with a continuous, violent choreic/ballistic movement of the left arm, leg, and trunk of 6 months' duration. At the beginning, she experienced vague discomfort in her left arm after a brief episode of heavy lifting. An involuntary choreic movement gradually developed in her arm within several hours. Owing to cramping pain associated with a flinging movement in her left arm and hand, she was initially treated with nonsteroidal anti-inflammatory drugs and physical therapy. However, the choreic movement progressively worsened over the following 


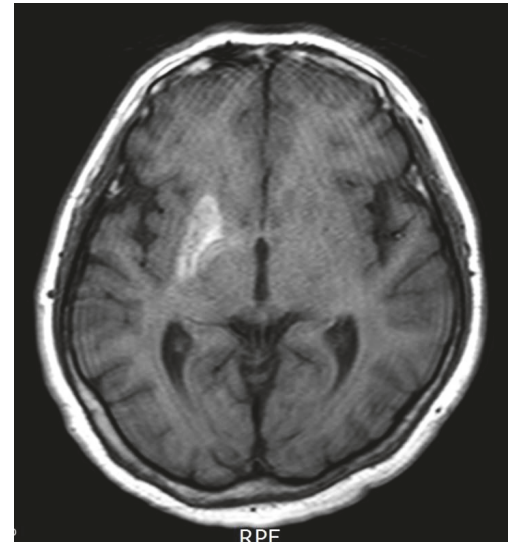

(a) A T1-weighted MRI image showing hyperintensity involving the right putamen and globus pallidus. The caudate nucleus was spared in this case

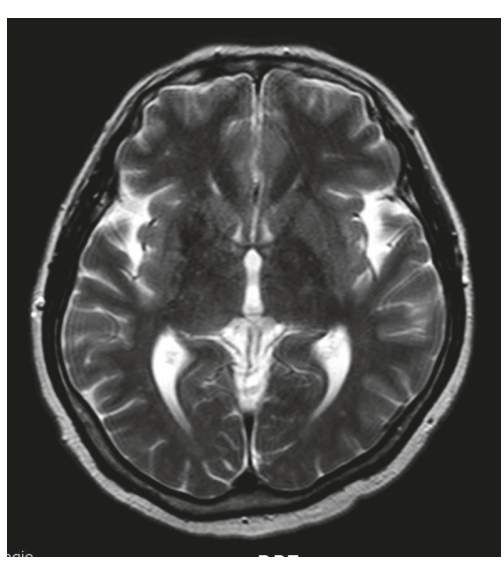

(b) An irregular area of hypointense lesion within the isointense putamen and globus pallidus was found on a T2-weighted MRI image

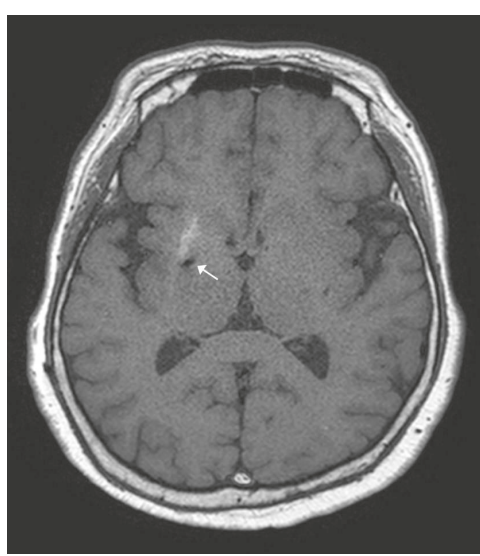

(c) A T1-weighted MR image taken 16 months after the onset of diabetic $\mathrm{HH}$. T1 hyperintensity in the right putamen was still identified but much attenuated, compared to the initial MRI. Note the hypointense metallic artefact of the DBS lead (arrow)

FIGURE 1: Magnetic resonance imaging (MRI) findings of diabetic hemichorea/hemiballism.

2 weeks and eventually became ballistic and involved her left arm and leg and had a high amplitude. She was admitted to another hospital via the emergency department for evaluation. Neurologic examination showed facial dyskinesis (grimacing) and a ballistic movement in her left trunk, arm, and legs. The movements could not be suppressed voluntarily but ceased during sleep. Muscle tone and strength in the upper and lower extremities were normal bilaterally. There was no sensory impairment and her cranial nerves were normal. Laboratory studies revealed a fasting blood glucose level of $536 \mathrm{mg} / \mathrm{dl}$, a serum osmolarity of $335 \mathrm{mOsm} / \mathrm{kg}$, and an $\mathrm{HbAlc}$ count of $15.7 \%$. Urinalysis was negative for ketones. There was no history of dopamine agonist or estrogen medication use or rheumatic fever/Sydenham's chorea.

A T1-weighted MRI image revealed a region of increased signal intensity restricted to the right putamen and globus pallidus, which was isointense on T2-weighted images (Figure 1). No abnormal enhancement with gadolinium was found. Under a diagnosis of nonketotic hyperglycemic hyperosmolar syndrome, the patient's blood glucose level was controlled with subcutaneous injection of insulin. The severe ballistic movement progressively improved with titration of haloperidol (up to $10 \mathrm{mg} /$ day), clonazepam (6 mg/day), amantadine $(300 \mathrm{mg} /$ day $)$, and tiapride $(200 \mathrm{mg} /$ day $)$ for $\mathrm{HH}$. Clinical manifestations progressively improved over the course of 3 months of DM control with neuroleptics but did not disappear. She suffered continuous $\mathrm{HH}$, which was most severe in the left leg and foot and involved a severe gait impairment. Finally the patient was referred to our clinic for surgical treatment for chronic, disabling $\mathrm{HH}$ at 6 months after the onset of diabetic $\mathrm{HH}$. Considering the chronicity and medical intractability of the situation, GPi DBS was decided upon.

The patient underwent a stereotactic MRI scan (1.5 tesla Archieva ${ }^{\circledR}$, Philips, Best, The Netherlands), and volumetric T1-weighted three-dimensional images, T2-weighted images, and proton density images were obtained. The images were transferred to a Framelink ${ }^{\circledR}$ planning station (version 4.1, Medtronic, Minneapolis, MN, USA) so that we could determine the coordinates of the image-guided GPi target and were reformatted for extraventricular trajectory planning (Figure 2(a)) [10, 11]. Implantation of the electrode (model 6149, St. Jude Medical, Plano, Texas, USA) was performed under $\mathrm{C}$-arm fluoroscopy guidance under the general anesthesia. After implantation of the lead, "O-arm" intraoperative CT scan (Medtronic, Minneapolis, MN, USA) was performed and the images were again transferred to the Framelink ${ }^{\circledR}$ planning station and merged with the preoperative volumetric T1-weighted MR images to verify the location of the electrodes (Figure 2(b)). After verification of electrode location, the distal end of the electrode was tunneled subcutaneously for external stimulation. After the patient recovered from anesthesia, an immediate postoperative computed tomography (CT, $1 \mathrm{~mm}$ thick axial slices) scan was taken again for verification of the location of the lead with the preoperative $\mathrm{T} 1$ MR images and intraoperative $\mathrm{O}$-arm images (Figure 2(c)).

A prominent mechanical effect with significant reduction (50\% reduction in intensity) of the choreic and proximal ballistic movements was observed. Bipolar electrical stimulation $(100-130 \mathrm{~Hz}, 1-3 \mathrm{~mA}, 130 \mathrm{usec}$, up to $3.5 \mathrm{~mA})$ further suppressed $\mathrm{HH}$, and the hyperkinetic movements became grossly undetectable after 7 days of external stimulation. No stimulation-related side effects such as dysarthria, dysphasia, or motor contraction were observed. A pulse generator (Libra ${ }^{\circledR}$, St. Jude Medical, Plano, TX, USA) was implanted via a transaxillary subpectoral route after 7 days of external stimulation [12]. The patient returned to her usual activities and became reemployed in her previous job on diabetic medication control and clonazepam ( $1.5 \mathrm{mg} /$ day). However, she felt continuous cramping pain and an internal sensation of muscular contraction in her lower leg and foot without any grossly visible choreic movements when walking. 


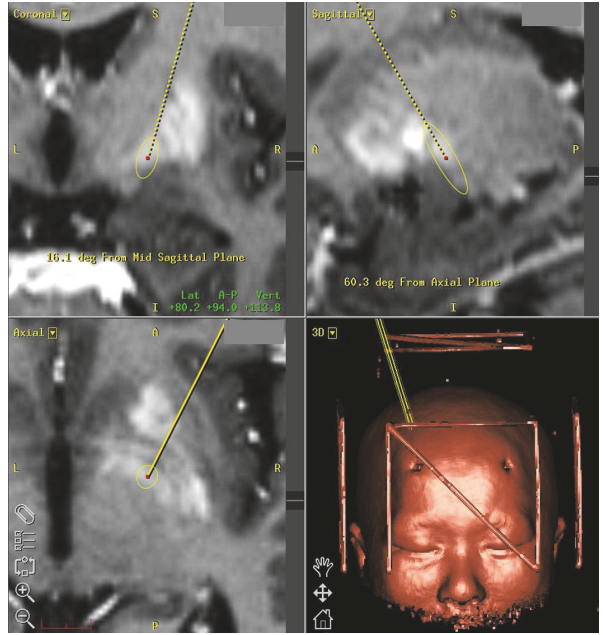

(a) An image in the planning software showing target localization and trajectory planning for right GPi DBS. The target was directed to the posterolateral portion of the GPi which was easily identified owing to hyperintensity in the volumetric T1-weighted MRI images

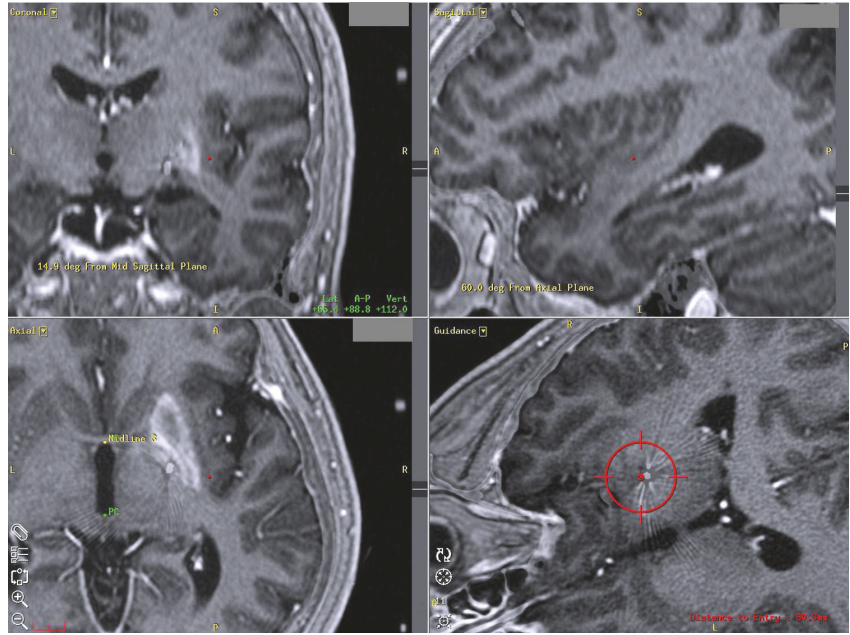

(b) An image captured from the planning software after fusion of an intraoperative $\mathrm{O}$-arm CT scan with preoperative volumetric $\mathrm{T} 1$ images to verify the location of the implanted lead, which was taken with the patient under general anesthesia. Although the electrode was found to be about $1 \mathrm{~mm}$ posterior and medial to the initial target point, it was accepted

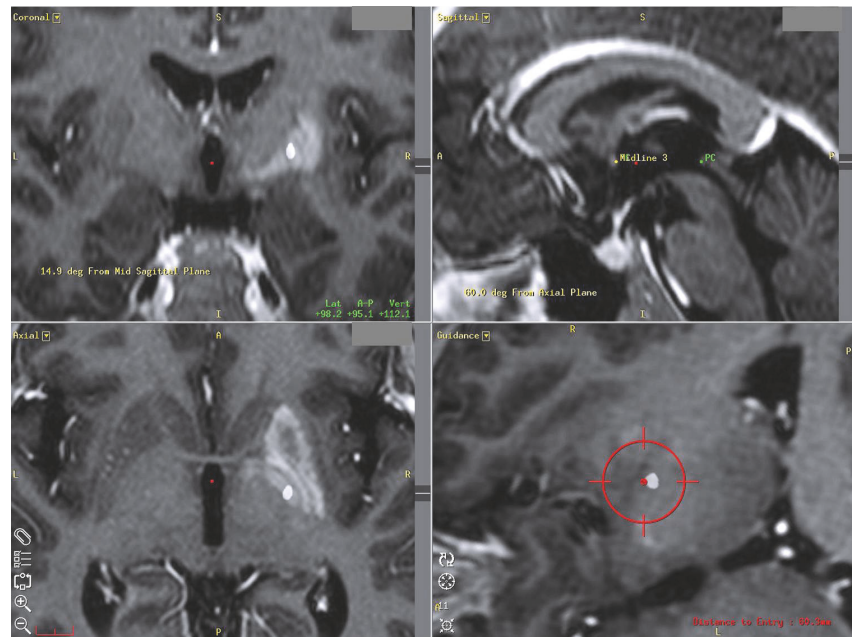

(c) An image captured from the planning software after fusion of the postoperative $\mathrm{CT}$ scan with preoperative volumetric $\mathrm{T} 1$ images. This image corresponds to the center of the 2nd contact (number 2), which was used as an anode for chronic stimulation

FIGURE 2: Image-guided deep brain stimulation (DBS) of the globus pallidus internus (GPi) for refractory diabetic $\mathrm{HH}$.

Chronic GPi stimulation was performed with regular adjustment of the stimulation parameters $(2.6-3.0 \mathrm{~mA}, 110-130 \mathrm{~Hz}$, 110-120 usec, $1(-), 2(+))$ every 3 months. At a 16-month postoperative follow-up, the patient was grossly free of $\mathrm{HH}$. However, minimal chorea in the left calf and foot was noted. She was readmitted for an on and off stimulation study and a follow-up MRI examination. After 30-minute off stimulation, the ballistic movements of the left thigh, distal leg, and shoulder returned and gait difficulty was noticed along with chorea in the left foot. After 10 minutes of on stimulation, the $\mathrm{HH}$ in her left leg and foot disappeared almost completely. Her fasting blood glucose, serum osmolarity, and HbAlc level were $109 \mathrm{mg} / \mathrm{dl}, 291 \mathrm{mOsm} / \mathrm{kg}$, and $7.9 \%$, respectively. T1weighted MRI images again revealed a high signal intensity confined to the right putamen, which was greatly attenuated compared with that seen on the initial preoperative MRI scan (Figures 1(a) and 1(c)).

\section{Discussion}

3.1. Diabetic HH. Transient chorea/ballism provoked by an episode of nonketotic hyperglycemia has repeatedly been reported over the past couple of decades and is the second most common cause of hemiballism [12]. The blood glucose level in patients with the condition ranges from 500 to $1000 \mathrm{mg} / \mathrm{dL}$ during the hyperglycemic precipitating crisis. $\mathrm{HH}$ is commonly associated with type $2 \mathrm{DM}$ and is rarely associated with type $1 \mathrm{DM}$. Although diabetic $\mathrm{HH}$ mostly 
TABLE 1: Summary of stereotactic surgery for diabetic hemichorea/hemiballism.

\begin{tabular}{|c|c|c|c|c|c|c|c|}
\hline Study/year & $\begin{array}{c}\text { Number of } \\
\text { patients }\end{array}$ & Age/sex & $\begin{array}{c}\text { Timing of } \\
\text { surgery }\end{array}$ & $\begin{array}{c}\text { Surgery } \\
\text { (lesion/DBS) }\end{array}$ & Results & $\begin{array}{l}\text { follow-up } \\
\text { period }\end{array}$ & Remarks \\
\hline Takamatsu et al. [6], 1995 & 1 & $57 / f$ & $1.5 \mathrm{mo}$. & VL lesion & Excellent & 4 years & \\
\hline Nakano et al. [8], 2005 & 1 & $65 / \mathrm{m}$ & $5 \mathrm{mo.}$ & $\begin{array}{c}\text { VO (Voa, Vop) } \\
\text { DBS }\end{array}$ & Effective & $9 \mathrm{mo}$. & Persistent $\mathrm{HH}$ in off stimulation \\
\hline Goto et al. [7], 2010 & 1 & $78 / f$ & N/A & GPi lesion & Excellent & $12 \mathrm{mo.}$ & HH immediately disappeared \\
\hline Current case, 2017 & 1 & $46 / f$ & $6 \mathrm{mo.}$ & GPi DBS & Effective & $16 \mathrm{mo.}$ & Persistent $\mathrm{HH}$ in off stimulation \\
\hline
\end{tabular}

GPi: globus pallidus internus; HH: hemichorea/hemiballism; mo: months; VL: ventral lateral nucleus; VO: ventralis oralis; Voa: ventralis oralis anterior; Vop: ventralis oralis posterior.

occurs when patients are in a state of nonketotic hyperglycemia, some patients also have ketotic hyperglycemia [13]. The movements are typically unilateral but can be generalized in rare cases [5]. Description of the movements ranges from mild chorea to severe ballism. According to a review of 53 cases of diabetic $\mathrm{HH}$ [4], the average age of onset is 71, and there is a female predominance of $1.8: 1$. Genetic factors may play a role, with an Asian predominance in reported cases [4].

High intensity in the basal ganglia on T1-weighted MRI is a characteristic finding in diabetic $\mathrm{HH}[2-6,14,15]$. T1 striatal hyperintensity involves the putamen in all cases, the head of the caudate nucleus in most cases, and the globus pallidus in a minority of cases $[14,15]$. In contrast to typical T1 hyperintensity, the findings on T2-weighted images are variable and range from hyper- and iso- to hypointensity. Gradient-echo and gadolinium-enhanced T1-weighted images are reported to be normal. Several mechanisms have been proposed to explain T1 striatal hyperintensity, including acute ischemia, petechial microhemorrhage, injury secondary to hyperviscosity, and vasogenic edema [14]. Currently, hyperviscosity is suggested to be the most plausible mechanism because of the following findings [14]: elevated serum osmolarity at the time of $\mathrm{HH}$, variable T2-weighted MR signal changes that reflect the difference in patterns and severity of hyperviscosity, and elevated myoinositol and choline levels seen on MR spectroscopy that are in line with a finding of abundant gemistocytes with increased protein content [14-17].

The putamen has been suggested to play a central role in diabetic $\mathrm{HH}$ [14]. The putamen has abundant medium-sized spiny neurons which store and release gamma amino butyric acid (GABA) [18], and there is universal involvement of the putamen in diabetic $\mathrm{HH}[14,15]$. Therefore, dysfunction of GABAergic neurons in the putamen may result in a disturbance of output nuclei of the basal ganglia, such as the substantia nigra and GPi [14]; resultant disinhibition of the motor thalamus ensues, leading to hyperkinetic movement disorder.

3.2. Treatment of Diabetic HH. Diabetic $\mathrm{HH}$ generally resolves over time, and often no treatment is necessary. Determining the etiology of the condition is a matter of paramount importance and correcting nonketotic hyperglycemia along with long-term diabetic control is the mainstay of treatment $[2,4,12]$. In cases of prolonged diabetic $\mathrm{HH}$, dopamine receptor blocking agents (chlorpromazine or haloperidol), neuroleptics (olanzapine, clozapine), and dopamine depleting agents (tetrabenazine) have been reported to be beneficial [2]. However, there are some rare patients who continue to have persistent $\mathrm{HH}$ years after the inciting hyperglycemic crisis [5]. The exact incidence of medically refractory diabetic $\mathrm{HH}$ is unclear. In rare, medically refractory diabetic $\mathrm{HH}$, stereotactic surgery has sporadically been reported in single case reports and we summarized them in Table 1.

Owing to the limited reports on surgical intervention against diabetic $\mathrm{HH}$, the timing and type of surgery, lesioning, or DBS are difficult to determine. However, despite the limited evidence, both lesioning and stimulation in the VL or VO thalamus and GPi showed good results [6-8]. Because diabetic $\mathrm{HH}$ is especially prevalent in Asians, all 3 cases regarding surgical treatment came from Japan [68]. We chose the GPi as a target of surgical intervention because previous reports regarding GPi lesioning produced uniformly positive results against diabetic $\mathrm{HH}$ [7] as well as $\mathrm{HH}$ secondary to vascular insults involving the STN and the brain stem [9, 19-21]. In addition, determination of the target within the GPi and trajectory planning was easily done with stereotactic MR images owing to prominent $\mathrm{T} 1$ hyperintensity in the GP (Figure 2(a)). A case of DBS of the VO thalamic nucleus was reported to be effective in diabetic $\mathrm{HH}$ [8], and we also achieved significant long-term effectiveness with GPi stimulation. Owing to the rarity of diabetic $\mathrm{HH}$, the present case is the first report of GPi DBS for refractory diabetic $\mathrm{HH}$. The present case showed persistent choreiform dyskinesia during off GPi stimulation even after 16 months following the onset of diabetic $\mathrm{HH}$ despite longterm continuous diabetic control and medical treatment with much attenuation of T1 hyperintensity in the follow-up MRI. Therefore, timely surgical intervention would be of help in alleviation of disabling motor symptoms in rare patient with persistent diabetic $\mathrm{HH}$.

\section{Conclusion}

We report the long-term effectiveness of GPi DBS in the treatment of chronic, disabling diabetic $\mathrm{HH}$.

\section{Conflicts of Interest}

The authors declare that they have no conflicts of interest regarding this manuscript. 


\section{References}

[1] J. Jankovic and S. Fahn, "Chorea, ballism, athetosis", in Principles and Practice of Movement Disorders, J. Jankovic and S. Fahn, Eds., pp. 401-403, Churchill Livingston Elsevier, Philadelphia, $\mathrm{Pa}$, USA, 1st edition, 2007.

[2] J. S. Hawley and W. J. Weiner, "Hemiballismus: current concepts and review," Parkinsonism \& Related Disorders, vol. 18, no. 2, pp. 125-129, 2012.

[3] T. Hashimoto, N. Hanyu, H. Yahikozawa, and N. Yanagisawa, "Persistent hemiballism with striatal hyperintensity on T1weighted MRI in a diabetic patient: A 6-year follow-up study," Journal of the Neurological Sciences, vol. 165, no. 2, pp. 178-181, 1999.

[4] S.-H. Oh, K.-Y. Lee, J.-H. Im, and M.-S. Lee, "Chorea associated with non-ketotic hyperglycemia and hyperintensity basal ganglia lesion on T1-weighted brain MRI studya meta-analysis of 53 cases including four present cases," Journal of the Neurological Sciences, vol. 200, no. 1-2, pp. 57-62, 2002.

[5] E. J. Ahlskog, H. Nishino, V. G. H. Evidente et al., "Persistent chorea triggered by hyperglycemic crisis in diabetics," Movement Disorders, vol. 16, no. 5, pp. 890-898, 2001.

[6] K. Takamatsu, T. Ohta, S. Sato et al., "Two diabetics with hemichorea hemiballism and striatal lesions," No To Shinkei, vol. 47, no. 2, pp. 167-172, 1995.

[7] T. Goto, T. Hashimoto, S. Hirayama, and K. Kitazawa, "Pallidal neuronal activity in diabetic hemichorea-hemiballism," Movement Disorders, vol. 25, no. 9, pp. 1295-1297, 2010.

[8] N. Nakano, T. Uchiyama, T. Okuda, M. Kitano, and M. Taneda, "Successful long-term deep brain stimulation for hemichoreahemiballism in a patient with diabetes," Journal of Neurosurgery, vol. 102, no. 6, pp. 1137-1141, 2005.

[9] H. Hasegawa, M. Samuel, J. Jarosz, and K. Ashkan, “The treatment of persistent vascular hemidystonia-hemiballismus with unilateral GPi deep brain stimulation," Movement Disorders, vol. 24, no. 11, pp. 1967-1968, 2009.

[10] B. C. Son, Y. M. Shon, J. G. Choi et al., "Clinical outcome of patients with deep brain stimulation of the centromedian thalamic nucleus for refractory epilepsy and location of the active contacts," Stereotactic and Functional Neurosurgery, vol. 94, no. 3, pp. 187-197, 2016.

[11] B. C. Son, S. H. Han, Y. S. Choi et al., "Transaxillary subpectoral implantation of implantable pulse generator for deep brain stimulation," Neuromodulation: Technology at the Neural Interface, vol. 15, no. 3, pp. 260-266, 2012.

[12] R. B. Postuma and A. E. Lang, "Hemiballism: revisiting a classic disorder," The Lancet Neurology, vol. 2, no. 11, pp. 661-668, 2003.

[13] Y. Abe, T. Yamamoto, T. Soeda et al., "Diabetic striatal disease: Clinical presentation, neuroimaging, and pathology," Internal Medicine, vol. 48, no. 13, pp. 1135-1141, 2009.

[14] N. Kandiah, K. Tan, C. C. T. Lim, and N. Venketasubramanian, "Hyperglycemic choreoathetosis: Role of the putamen in pathogenesis," Movement Disorders, vol. 24, no. 6, pp. 915-919, 2009.

[15] C. Battisti, F. Forte, E. Rubenni et al., "Two cases of hemichoreahemiballism with nonketotic hyperglycemia: A new point of view," Neurological Sciences, vol. 30, no. 3, pp. 179-183, 2009.

[16] K. Chu, D. W. Kang, D. E. Kim, S. H. Park, and J. K. Roh, "Diffusion-weighted and gradient echo magnetic resonance findings of hemichorea-hemiballismus associated diabetic hyperglycemia: a hyperviscosity syndrome?" Archives of Neurology, vol. 59, no. 3, pp. 448-452, 2003.
[17] D. E. Shan, D. M. Ho, C. Chang, H. C. Pan, and M. M. Teng, "Hemichorea-hemiballismus: an explanation for MR signal changes," American Journal of Neuroradiology, vol. 19, pp. 863870, 1998.

[18] A. M. Graybiel, "Neurotransmitters and neuromodulators in the basal ganglia," Trends in Neurosciences, vol. 13, no. 7, pp. 244254, 1990.

[19] J. I. Suarez, L. Verhagen Merman, S. G. Reich, P. M. Dougherty, M. Hallett, and F. A. Lenz, "Pallidotomy for hemiballismus: Efficacy and characteristics of neuronal activity," Annals of Neurology, vol. 42, no. 5, pp. 807-811, 1997.

[20] J. L. Vitek, V. Chockkan, J.-Y. Zhang et al., "Neuronal activity in the basal ganglia in patients with generalized dystonia and hemiballismus," Annals of Neurology, vol. 46, no. 1, pp. 22-35, 1999.

[21] K. Yamada, M. Harada, and S. Goto, "Response of postapoplectic hemichorea/ballism to GPi pallidotomy: Progressive improvement resulting in complete relief," Movement Disorders, vol. 19, no. 9, pp. 1111-1114, 2004. 


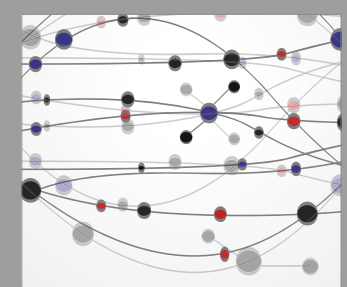

The Scientific World Journal
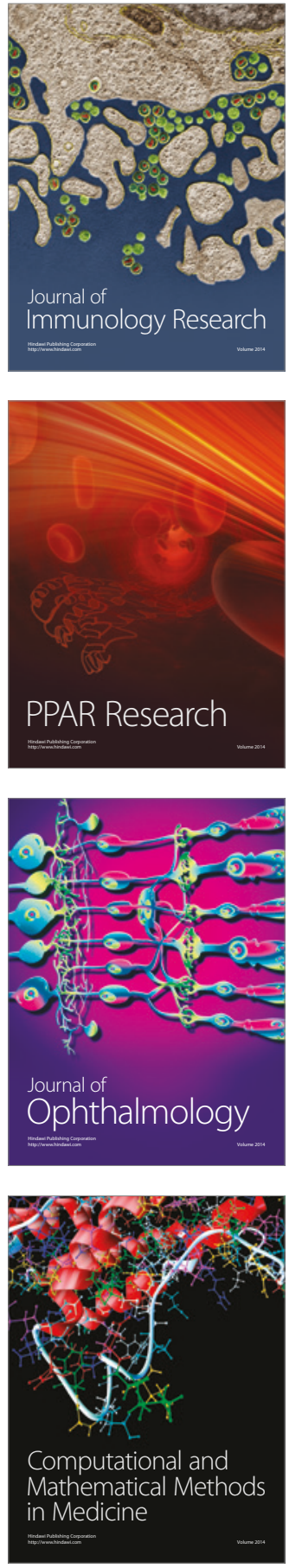

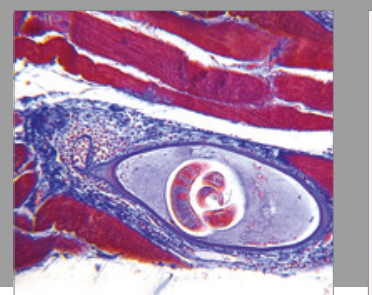

Gastroenterology Research and Practice
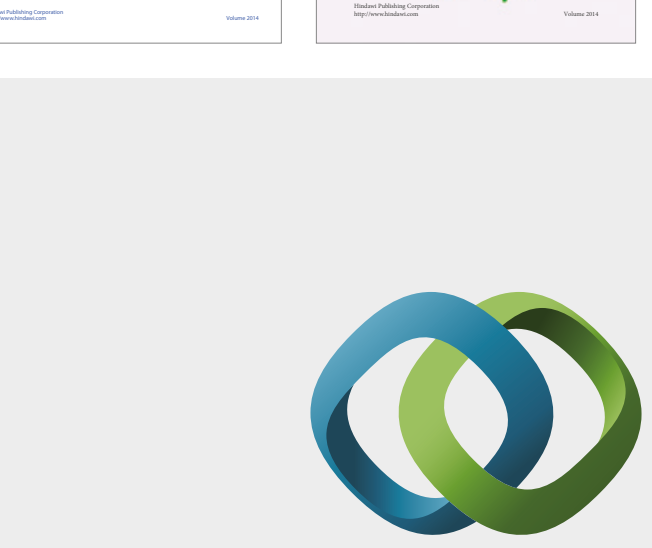

\section{Hindawi}

Submit your manuscripts at

https://www.hindawi.com
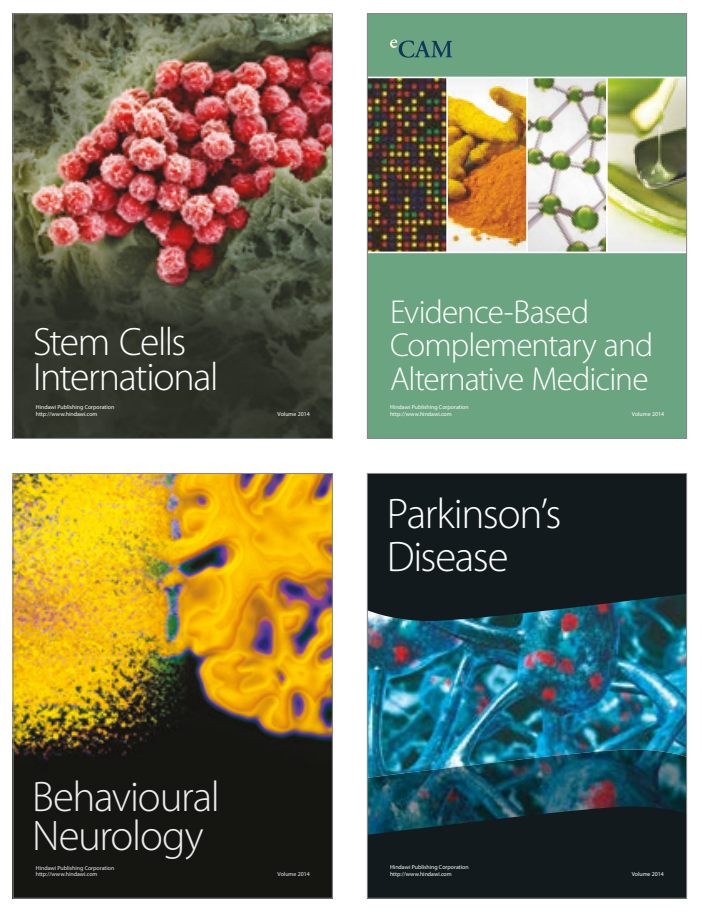
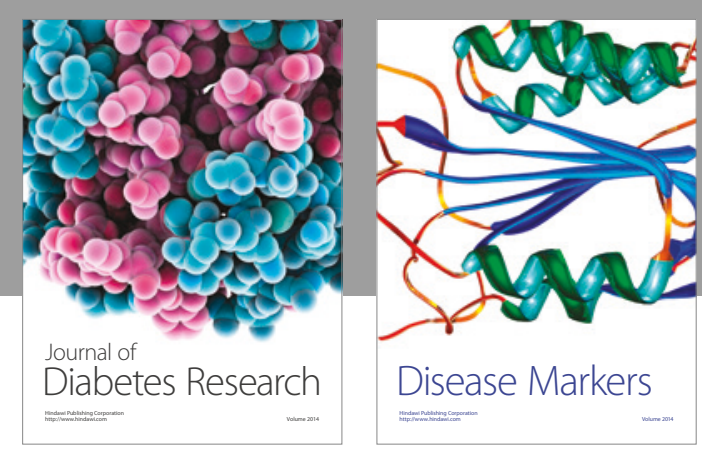

Disease Markers
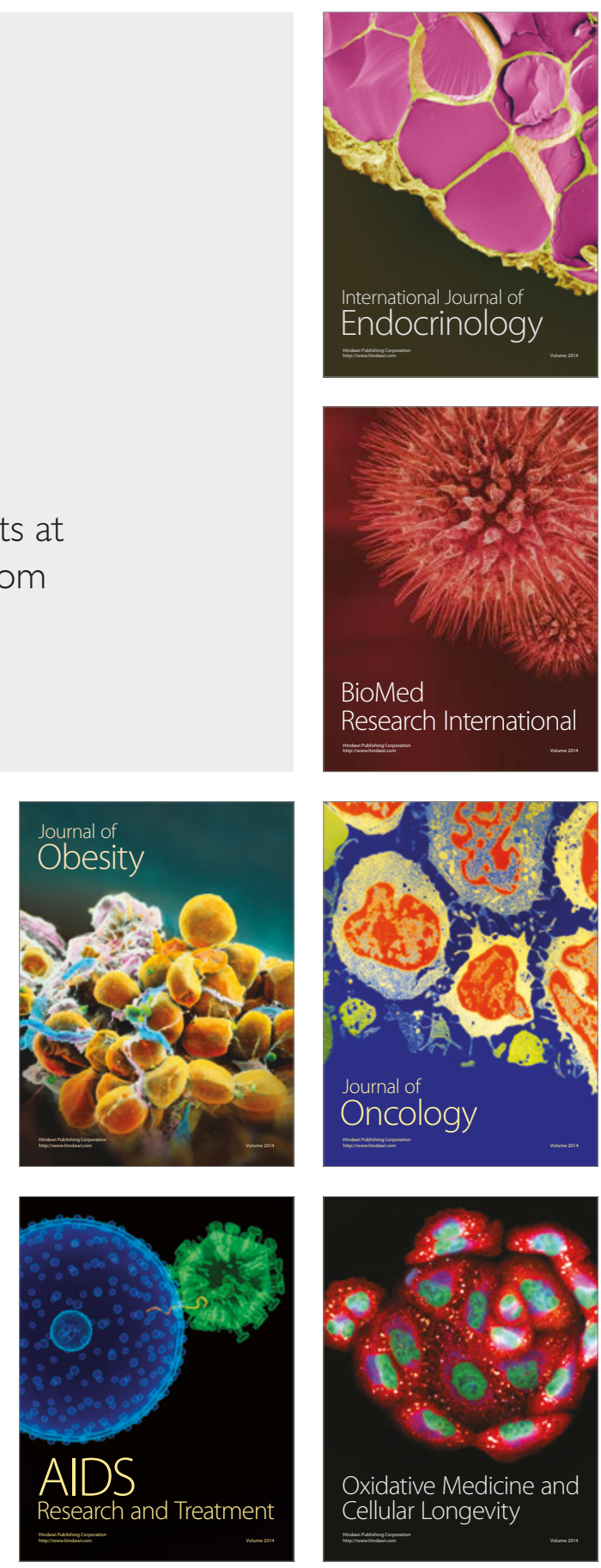